Trinity University

Digital Commons @ Trinity

Mathematics Faculty Research

Mathematics Department

$10-2010$

\title{
Non-Autonomous Periodic Systems with Allee Effects
}

Rafael Luís

Saber Elaydi

Trinity University, selaydi@trinity.edu

Henrique Oliveira

Follow this and additional works at: https://digitalcommons.trinity.edu/math_faculty

Part of the Mathematics Commons

\section{Repository Citation}

Luís, R., Elaydi, S., \& Oliveira, H. (2010). Non-autonomous periodic systems with Allee effects. Journal of Difference Equations and Applications, 16(10), 1179-1196. http://doi.org/10.1080/10236190902794951

This Post-Print is brought to you for free and open access by the Mathematics Department at Digital Commons @ Trinity. It has been accepted for inclusion in Mathematics Faculty Research by an authorized administrator of Digital Commons @ Trinity. For more information, please contact jcostanz@trinity.edu. 


\title{
Nonautonomous periodic systems with Allee effects
}

\author{
Rafael Luís ${ }^{\mathrm{a}} \dagger$, Saber Elaydi ${ }^{\mathrm{b}} \S$ and Henrique Oliveira ${ }^{\mathrm{c}} \boldsymbol{\uparrow *}$ \\ ${ }^{a}$ Center for Mathematical Analysis, Geometry, and Dynamical Systems, Instituto \\ Superior Técnico, Technical University of Lisbon, Portugal; ${ }^{\mathrm{b}}$ Department of \\ Mathematics, Trinity University, San Antonio, Texas, USA; ${ }^{\mathrm{c}}$ Department of \\ Mathematics, Instituto Superior Técnico, Technical University of Lisbon, Portugal
}

(Received)

\begin{abstract}
A new class of maps called unimodal Allee maps are introduced. Such maps arise in the study of population dynamics in which the population goes extinct if its size falls below a threshold value. A unimodal Allee map is thus a unimodal map with tree fixed points, a zero fixed point, a small positive fixed point, called threshold point, and a bigger positive fixed point, called the carrying capacity. In this paper the properties and stability of the three fixed points are studied in the setting of nonautonomous periodic dynamical systems or difference equations. Finally we investigate the bifurcation of periodic systems/difference equations when the system consists of two unimodal Allee maps.
\end{abstract}

Keywords: Unimodal Allee maps, Threshold point, Carrying capacity, Composition map, Stability, Bifurcation.

\section{Introduction}

The Allee effect is a phenomena in population dynamics attributed to the biologist Wander Claude Allee [1]. Allee proposed that the per capita birth rate declines at low density or population sizes. In the languages of dynamical systems or difference equations, a map representing the Allee effect must have tree fixed points, an asymptotically stable zero fixed point, a small unstable fixed point, called the threshold point, and a bigger positive fixed point, called the carrying capacity, that is asymptotically stable at least for smaller values of the parameters.

Recently, there has been a surge in research activities on models with Allee effect and a publication of a book dedicated solely to this phenomenon [5].

Some of the relevant work may be found in Yakubu [15, 16], Jang [21], Li, Song, and Wang [12], Elaydi and Sacker [17], Allen, Fagan, Hognas, and Fagerholm [2], Luís, Elaydi and Oliveira [13], Schreiber [19], Dennis [6] and Cushing [7].

Our main interest in this paper is to study nonautonomous periodic difference equations/discrete dynamical systems in which the maps of the system are unimodal Allee maps. Such systems model population with fluctuating habitat and they are commonly called periodically forced systems.

\footnotetext{
†Corresponding author. Email: rafaelluis@netmadeira.com

$\S$ Email: selaydi@trinity.edu

ๆEmail: holiv@math.ist.utl.pt

* This work is part of the first author's Ph.D. dissertation
} 


\section{Preliminaries}

Consider the set $\mathcal{F}=\left\{f_{0}, f_{1}, \ldots, f_{p-1}\right\}$ of continuous maps on $I=[0, b]$, where $b \leq \infty$. The set $\mathcal{F}$ generates the nonautonomous $p$-periodic difference equation

$$
x_{n+1}=f_{n}\left(x_{n}\right), n \in \mathbb{Z}^{+},
$$

where $\mathbb{Z}^{+}:=\{0,1,2,3, \ldots\}$ and $f_{n+p}=f_{n}, \forall n \in \mathbb{Z}^{+}$.

Though the nonautonomous periodic difference equation (1) does not generate a discrete dynamical system [9], one may speak about the nonautonomous $p$-periodic dynamical system $\mathcal{F}$. One of the most effective way of converting the nonautonomous difference equation (1) into a genuine discrete dynamical system is the construction of the associated skew-product system as described a recent paper by Elaydi and Sacker [10]. It is noteworthy to mention that this idea was originally used to study nonautonomous differential equations by Sacker and Sell [18]. However, since the focus here will be on the case when $\mathcal{F}$ consists of two maps, we will not utilize the skew-product construction as it is more appropriate for more complicated setting.

We now present few basic definitions that will be used in the sequel.

Definition 2.1. A point $x^{*}$ is a fixed point of Eq. (1) or the systems $\mathcal{F}$ if $f_{n}\left(x^{*}\right)=$ $x^{*}$ for all $n \in \mathbb{Z}^{+}$. In other words, $x^{*}$ is a fixed point of all the maps in $\mathcal{F}$.

Definition 2.2. Let $C_{r}=\left\{\bar{x}_{0}, \bar{x}_{1}, \ldots, \bar{x}_{r-1}\right\}$ be a ordered set in $I$. Then $C_{r}$ is called an periodic $r$-cycle (orbit) if

$$
f_{(i+n r) \operatorname{modp}}\left(\bar{x}_{i}\right)=\bar{x}_{(i+1) \operatorname{modr}}, 0 \leq i \leq r-1 .
$$

To this end we have talked about general continuous maps on an interval. The focus in this paper will be on special types of map that we call unimodal Allee maps. A definition of these maps now follows.

Definition 2.3. Let $I=[0, b] \subset \mathbb{R}^{+}$. A continuous function $f: I \rightarrow I$ is called an Allee map if the following hold:

- $f(0)=0$, and there are positive points $A_{f}$ and $K_{f}$ such that

- $f(x)<x$ for $x \in\left(0, A_{f}\right) \cup\left(K_{f}, b\right)$ and $f(x)>x$ for $x \in\left(A_{f}, K_{f}\right)$.

If, in addition, the map is unimodal, then it is called an unimodal Allee map. Explicitly, we require the following:

- $f(b)=0$ when $b$ is finite or $\lim _{x \rightarrow+\infty} f(x)=0$ otherwise.

- There exists a unique critical point $C_{f}$ of $f$, where $f(x)$ is strictly increasing on $\left[0, C_{f}\right)$ and strictly decreasing on $\left(C_{f}, b\right)$ (or $\left(C_{f},+\infty\right)$ when $\left.b=+\infty\right)$.

Fig. 1 depicts a prototype of unimodal Allee maps.

From definition 2.3, it follows that $A_{f}$ and $K_{f}$ are positive fixed points. We call the smaller positive fixed point the threshold point $A_{f}$ of the map $f$, and the greater positive fixed point $K_{f}$ the carrying capacity of the map $f$. It is easy to verify that $x^{*}=0$ is an attracting fixed point and $\left[0, A_{f}\right) \cup\left(\tilde{A}_{f}, \infty\right) \subset \mathcal{B}_{f}(0)$, where $\mathcal{B}_{f}(0)$ is the basin of attraction of zero and $\tilde{A}_{f}=f^{-1}\left(A_{f}\right)$, i.e. $f\left(\tilde{A}_{f}\right)=A_{f}$, with $\tilde{A}_{f}>K_{f}$. Note that the threshold point $A_{f}$ is always repelling while the carrying 


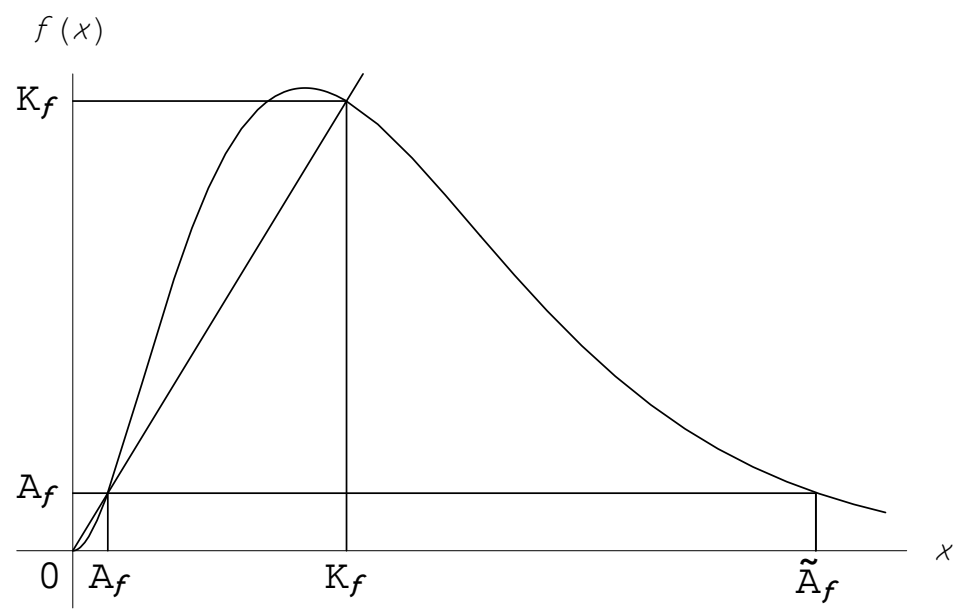

Figure 1. An instance of one unimodal Allee map $f$

capacity $K_{f}$ may or not stable.

Next we define a unimodal Allee map $f$ by two maps, a left map $f_{l}$ and a right $\operatorname{map} f_{r}$.

Thus we have

$$
f(x)=\left\{\begin{array}{l}
f_{l}(x) \text { if } 0 \leq x \leq A_{f} \\
f_{r}(x) \text { if } A_{f}<x \leq b
\end{array} .\right.
$$

It follows that $f(0)=f_{l}(0)=f_{r}(b)=0$ (or $\lim _{x \rightarrow \infty} f_{r}(x)=0$ ). Since $f$ is continuous in $\mathbb{R}^{+}$, it follows that $f\left(A_{f}\right)=f_{l}\left(A_{f}\right)=f_{r}\left(A_{f}\right)=A_{f}$ and $f\left(K_{f}\right)=f_{r}\left(K_{f}\right)=K_{f}$.

To facilitate our study we introduce two zones, the threshold zone and the carrying capacity zone.

\section{Definition 2.4.}

(1) The square that contains the origin and the points $\left(A_{f}, 0\right),\left(0, A_{f}\right)$ and $\left(A_{f}, A_{f}\right)$ will be called the threshold zone.

(2) The rectangle that contains the points $\left(A_{f}, A_{f}\right),\left(A_{f}, f\left(C_{f}\right)\right),\left(\tilde{A}_{f}, A_{f}\right)$ and $\left(\tilde{A}_{f}, f\left(C_{f}\right)\right)$ will be called the carrying capacity zone.

Consider now the nonautonomous periodic system $\mathcal{W}=\{f, g\}$ where $f$ and $g$ are unimodal Allee maps with $f(x)>g(x)$ for all $x$ on $(0, b)$. We note that under this hypothesis, we have $0<A_{f}<A_{g}<K_{g}<K_{f}$. Henceforth we assume that the right end point $b$ of $I$ is fixed for all the unimodal Allee maps.

The composition map $f \circ g$ may be written as follows

$$
f \circ g=\left\{\begin{array}{l}
f\left(g_{l}(x)\right) \text { if } 0 \leq x \leq A_{g} \\
f\left(g_{r}(x)\right) \text { if } A_{g}<x \leq b
\end{array} .\right.
$$

The first branch of (3) may be written as

$$
f\left(g_{l}(x)\right)=\left\{\begin{array}{l}
f_{l}\left(g_{l}(x)\right) \text { if } 0 \leq g_{1}(x) \leq A_{f} \wedge 0 \leq x \leq A_{g} \\
f_{r}\left(g_{l}(x)\right) \text { if } A_{f} \leq g_{1}(x)<b \wedge 0 \leq x \leq A_{g}
\end{array}\right.
$$


hence

$$
f\left(g_{l}(x)\right)=\left\{\begin{array}{l}
f_{l}\left(g_{l}(x)\right) \text { if } 0 \leq x \leq A_{f}^{-} \\
f_{r}\left(g_{l}(x)\right) \text { if } A_{f}^{-}<x \leq A_{g}
\end{array},\right.
$$

where $A_{f}^{-}$represents the left preimage of $A_{f}$ under the map $g$, i.e., $g\left(A_{f}^{-}\right)=A_{f}$, or equivalently

$$
f_{l}\left(g_{l}\left(A_{f}^{-}\right)\right)=f_{r}\left(g_{l}\left(A_{f}^{-}\right)\right)=f\left(A_{f}\right)=A_{f} .
$$

The second branch of (3) may be written as

$$
f\left(g_{r}(X)\right)=\left\{\begin{array}{l}
f_{r}\left(g_{r}(x)\right) \text { if } A_{g}<x<A_{f}^{+} \\
f_{l}\left(g_{r}(x)\right) \text { if } A_{f}^{+} \leq x \leq b
\end{array}\right.
$$

where $A_{f}^{+}$represents the right preimage of $A_{f}$ under the map $g$, i.e., $g\left(A_{f}^{+}\right)=A_{f}$, or equivalently

$$
f_{r}\left(g_{r}\left(A_{f}^{+}\right)\right)=f_{l}\left(g_{r}\left(A_{f}^{+}\right)\right)=f\left(A_{f}\right)=A_{f} .
$$

From (4) and (5) we obtain

$$
f \circ g=\left\{\begin{array}{l}
f_{l}\left(g_{l}(x)\right) \text { if } 0 \leq x \leq A_{f}^{-} \\
f_{r}\left(g_{l}(x)\right) \text { if } A_{i}^{-}<x \leq A_{g} \\
f_{r}\left(g_{r}(x)\right) \text { if } A_{g}<x<A_{f}^{+} \\
f_{l}\left(g_{l}(x)\right) \text { if } A_{f}^{+} \leq x \leq b
\end{array}\right.
$$

Similarly

$$
g \circ f=\left\{\begin{array}{l}
g_{l}\left(f_{l}(x)\right) \text { if } 0 \leq x \leq A_{f} \\
g_{l}\left(f_{r}(x)\right) \text { if } A_{f}<x<A_{g}^{-} \\
g_{r}\left(f_{r}(x)\right) \text { if } A_{g}^{-} \leq x \leq A_{g}^{+} \\
g_{l}\left(f_{r}(x)\right) \text { if } A_{g}^{+}<x \leq b
\end{array}\right.
$$

where $A_{g}^{-}$and $A_{g}^{+}$represents the left and the right preimages of $A_{g}$ under the map $f$, respectively, that is, $f\left(A_{g}^{-}\right)=f\left(A_{g}^{+}\right)=A_{g}$. In other words we have

$$
g_{l}\left(f_{r}\left(A_{g}^{-}\right)\right)=g_{r}\left(f_{r}\left(A_{g}^{-}\right)\right)=g\left(A_{g}\right)=A_{g}
$$

and

$$
g_{r}\left(f_{r}\left(A_{g}^{+}\right)\right)=g_{l}\left(f_{r}\left(A_{g}^{+}\right)\right)=g\left(A_{g}\right)=A_{g} .
$$

Figure 2 summarises above remarks.

Lemma 2.5. Let $f, g \in \mathcal{W}$. If $C_{f}>A_{g}$, where $C_{f}$ is the unique critical point of $f$ and $A_{g}$ is the threshold point of $g$, then $f$ and $g$, both, are homeomorphisms on $\left[0, A_{g}\right]$.

\section{Threshold points of the composition map}

In this section we prove the existence of the fixed points, called threshold points, of the composition map. In addition we establish an order relation between these 

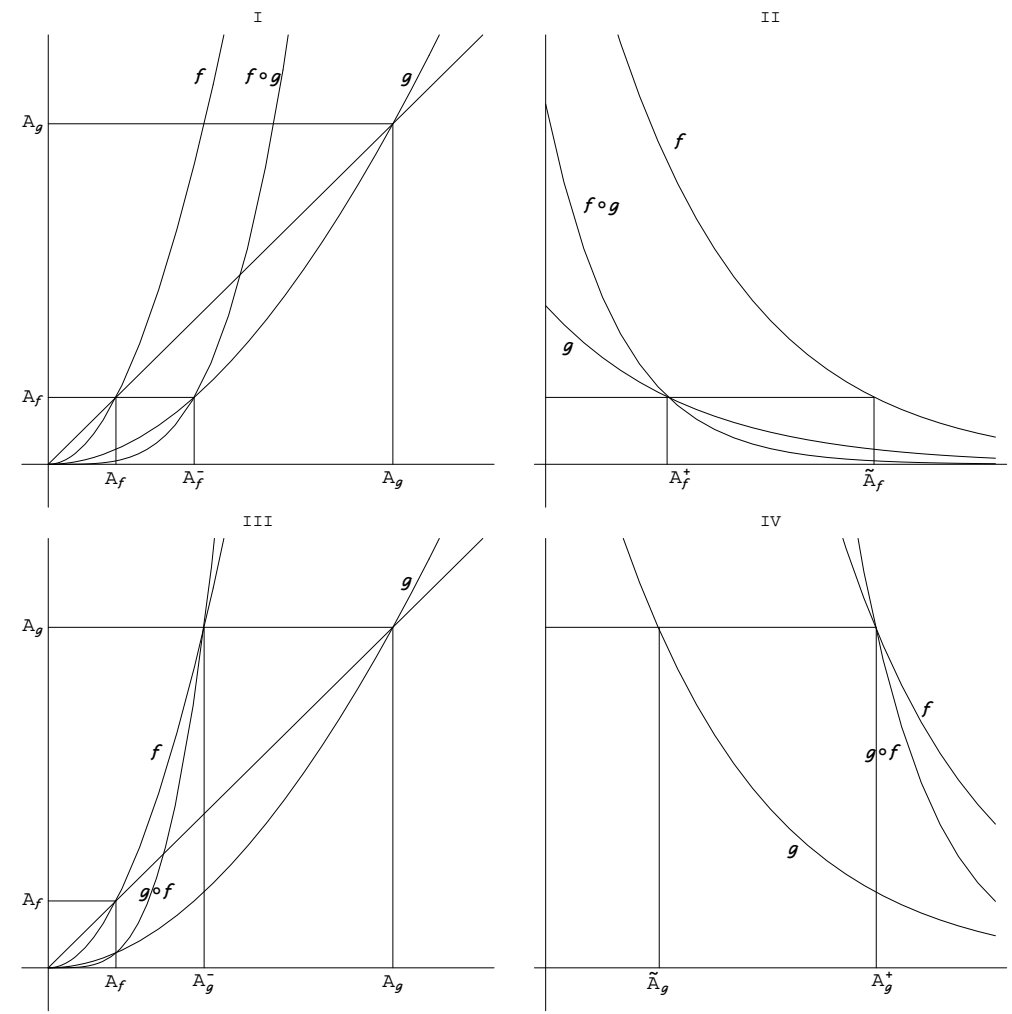

Figure 2. Parts I and II depicts the left and the right preimages of $A_{f}$ under $g$ while parts III and IV depicts the left and the right preimage of $A_{g}$ under the map $f$

fixed points.

From here to the rest of the paper we assume that $A_{f}$ and $A_{g}$ are the threshold points of the unimodal Allee maps $f$ and $g$, respectively. We also assume that $A_{f}^{-}$and $A_{g}^{-}$are, respectively, the first preimage of $A_{f}$ by the map $g$ and the first preimage of $A_{g}$ by the map $f$.

THEOREM 3.1. Let $f$ and $g$ be two unimodal Allee maps such that $f(x)>g(x)$ for all $x$ on $(0, b)$. Then both $f \circ g$ and $g \circ f$, have threshold points, that we denote by $A_{f g}$ and $A_{g f}$, respectively. Moreover $A_{f}^{-}<A_{f g}<A_{g}$ and $A_{f}<A_{g f}<A_{g}^{-}$.

Proof. Assume that $f$ and $g$ satisfy the conditions of the hypothesis of the theorem. First let us to prove the existence of $A_{f g}$ and $A_{g f}$. We know that $g_{l}\left(A_{f}\right)<A_{f}$ and on $\left[0, A_{f}\right] f$ is increasing. This implies that $f_{l}\left(g_{l}\left(A_{f}\right)\right)<f_{l}\left(A_{f}\right)=A_{f}$. On the other hand $f_{r}\left(g_{l}\left(A_{g}\right)\right)=f_{r}\left(A_{g}\right)>g_{l}\left(A_{g}\right)=A_{g}$. Hence the function $f \circ g(x)-x$ changes sign on $\left(A_{f}, A_{g}\right)$. Then there exits $x \in\left(A_{f}, A_{g}\right)$ such that $f \circ g(x)=x$, i.e. $A_{f}<A_{f g}<A_{g}$. In the same way we prove that $A_{f}<A_{g f}<A_{g}$.

To proof that $A_{f g} \in\left(A_{f}^{-}, A_{g}\right)$ first we will proof that $A_{f g} \notin\left[A_{f}, A_{f}^{-}\right]$. Let $x \in\left[A_{f}, A_{f}^{-}\right]$. We know that $f \circ g\left(A_{f}\right)<A_{f}$ and $f \circ g\left(A_{f}^{-}\right)=A_{f}<A_{f}^{-}$. When if $x \in\left(A_{f}, A_{f}^{-}\right)$we have that $g_{l}(x)<A_{f}$ and so $f_{l}\left(g_{l}(x)\right)<A_{f}<x$. Therefore $A_{f g} \notin\left[A_{f}, A_{f}^{-}\right]$.

Now let $x \in\left(A_{f}^{-}, A_{g}\right)$. By one side we have $f \circ g\left(A_{g}\right)=f\left(A_{g}\right)>A_{g}>x$ and on the other side $f \circ g\left(A_{f}^{-}\right)=A_{f}<x$, consequently, there exits $y \in\left(A_{f}^{-}, A_{g}\right)$ such that $f \circ g(y)=y$, that is, $A_{f g} \in\left(A_{f}^{-}, A_{g}\right)$. Following the same reasoning we prove $A_{f}<A_{g f}<A_{g}^{-}$.

Next we establish an order relation between these two threshold points of $f \circ g$ 
and $g \circ f$, respectively.

TheOREM 3.2. Let $f$ and $g$ be two unimodal Allee maps such that $f(x)>g(x)$ for all $x$ on $(0, b)$. Suppose that in the threshold region, i.e., on $J=\left[0, A_{g}\right]$, these two maps are convex, $f$ is increasing and $f^{\prime}(x)>g^{\prime}(x), \forall x \in J$. Suppose also that

$$
f^{\prime}\left(A_{g}\right)+g^{\prime}\left(A_{g}\right) \leq f^{\prime}\left(A_{f}\right) g^{\prime}\left(A_{g}\right) .
$$

Then $A_{g}^{-} \leq A_{f}^{-}$. Moreover $A_{g f}<A_{f g}$.

Proof. By hypothesis we have $A_{g}-A_{f}=\varepsilon>0$ and $\forall x \in J=\left[0, A_{g}\right], f(x)-g(x)=$ $\delta(x)>0$ such that $\delta(x)$ is increasing. We need to prove that the first preimage of $A_{f}$ and $A_{g}$, both, satisfy the relation $A_{g}^{-} \leq A_{f}^{-}$or $f^{-1}\left(A_{g}\right) \leq g^{-1}\left(A_{f}\right)$, that is equivalent

$$
A_{g} \leq f \circ g^{-1}\left(A_{f}\right)
$$

By the Taylor's series we know that

$$
\begin{aligned}
g^{-1}\left(A_{f}\right) & =g^{-1}\left(A_{g}-\varepsilon\right) \\
& =g^{-1}\left(A_{g}\right)-g^{-1^{\prime}}\left(A_{g}\right) \varepsilon+O\left(\varepsilon^{2}\right) \\
& =A_{g}-\frac{\varepsilon}{g^{\prime}\left(A_{g}\right)}+O\left(\varepsilon^{2}\right) .
\end{aligned}
$$

Substituting the previous relation in (9) we get

$$
A_{g} \leq f\left(A_{g}-\frac{\varepsilon}{g^{\prime}\left(A_{g}\right)}+O\left(\varepsilon^{2}\right)\right)
$$

and again by Taylor's series

$$
A_{g}=f\left(A_{g}\right)-\frac{f^{\prime}\left(A_{g}\right)}{g^{\prime}\left(A_{g}\right)} \varepsilon+O\left(\varepsilon^{2}\right)
$$

that is

$$
\frac{f^{\prime}\left(A_{g}\right)}{g^{\prime}\left(A_{g}\right)} \varepsilon \leq f\left(A_{g}\right)-A_{g}+O\left(\varepsilon^{2}\right) .
$$

Once that $f\left(A_{g}\right)-A_{g}=f\left(A_{g}\right)-g\left(A_{g}\right)=\delta$ we get

$$
\frac{f^{\prime}\left(A_{g}\right)}{g^{\prime}\left(A_{g}\right)} \varepsilon \leq \delta+O\left(\varepsilon^{2}\right) \approx \delta .
$$

So relation (9) is equivalent to relation (10).

$f$ is convex and therefore

$$
f^{\prime}\left(A_{f}\right)<\frac{f\left(A_{g}\right)-f\left(A_{f}\right)}{A_{g}-A_{f}}=f^{\prime}(M)<f^{\prime}\left(A_{g}\right),
$$

where $M \in] A_{f}, A_{g}\left[\right.$. So $f^{\prime}(M)=\frac{f\left(A_{g}\right)-A_{g}+A_{g}-A_{f}}{\varepsilon}$, and therefore $f^{\prime}(M)=\frac{\delta+\varepsilon}{\varepsilon}$.

By hypothesis we have $f^{\prime}\left(A_{g}\right)+g^{\prime}\left(A_{g}\right) \leq f^{\prime}\left(A_{f}\right) g^{\prime}\left(A_{g}\right)$, that is equivalent to $\frac{f^{\prime}\left(A_{g}\right)}{g^{\prime}\left(A_{g}\right)}+1 \leq f^{\prime}\left(A_{f}\right)$. But $f^{\prime}\left(A_{f}\right)<f^{\prime}(M)$ so

$$
\frac{f^{\prime}\left(A_{g}\right)}{g^{\prime}\left(A_{g}\right)}+1 \leq f^{\prime}(M)=\frac{\delta+\varepsilon}{\varepsilon}
$$

Multiplying by $\varepsilon$ both of the members of the last relation we get

$$
\frac{f^{\prime}\left(A_{g}\right)}{g^{\prime}\left(A_{g}\right)} \varepsilon \leq \delta
$$


that is equivalent to relation (10), and therefore this part of the theorem is proved.

From Theorem 3.1 and by the fact that $A_{g}^{-} \leq A_{f}^{-}$it follows $A_{g f}<A_{f g}$.

Hypothesis (8) requires that $f$ and $g$ stay sufficiently far apart to avoid the collapse of the interval where the threshold points of $f \circ g$ and $g \circ f$ belongs.

\section{The carrying capacity of the composition map}

In this section we study the existence, the location and the properties of the carrying capacity of the composition map.

Note that if $f$ and $g$ are two unimodal Allee maps such that $f(x)>g(x)$ for all $x$ on $(0, b)$, then the critical points of $f \circ g$ are the solutions of the equation $f^{\prime}(g(x)) g^{\prime}(x)=0$. This implies that $C_{g}$ is a critical point of both $g$ and $f \circ g$. The equation $f^{\prime}(g(x))=0$ has a solution if and only if the equation $g(x)=C_{f}$ has a solution. Thus either $g^{-1}\left(C_{f}\right)=\emptyset$ or $g^{-1}\left(C_{f}\right)$ consists of two points one on the left side of $C_{g}$ and the other on the right side of $C_{g}$. Let us to represent the points by $C_{f g}^{-}$(resp. $C_{f g}^{+}$), the critical point of the composition map $f \circ g$ on the left (resp. on the right) side of $C_{g}$.

So if $C_{f g}^{-}$and $C_{f g}^{+}$exists then the composition map $f \circ g$ has four intervals of monotonicity (otherwise $f \circ g$ has two intervals of monotonnicity). Explicitly, $f \circ g$ is strictly increasing on $\left[0, C_{f g}^{-}\right] \cup\left[C_{g}, C_{f g}^{+}\right]$and is strictly decreasing on $\left[C_{f g}^{-}, C_{g}\right] \cup\left[C_{f g}^{+}, b\right]$. The same analysis can be made for the map $g \circ f$.

Note that the threshold point of the composition map $f \circ g$ (resp. $g \circ f$ ) belongs always to the first interval where the composition map is increasing.

Recall from the previous sections that $K_{f}$ and $K_{g}$ are the carrying capacities of $f$ and $g$, respectively, and $A_{f}^{+}$(resp. $A_{g}^{+}$) the right positive preimage of $A_{f}$ (resp. $A_{g}$ ) under the map $g$ (resp. $f$ ). We also follows the notation about the critical points of the composition map that we described above.

TheOREM 4.1. Let $f$ and $g$ be two unimodal Allee maps such that $f(x)>g(x)$ for all $x$ on $(0, b)$. Then both $f \circ g$ and $g \circ f$, have carrying capacities, that we denote by $K_{f g}$ and $K_{g f}$, respectively. Moreover $K_{g}<K_{f g}<A_{f}^{+}$and $A_{g}^{-}<K_{g f}<A_{g}^{+}$.

Proof. It is clear that $0<A_{f}<A_{g}<K_{g}<K_{f}, A_{f}^{+}>K_{f}$ and $A_{g}^{-}<A_{g}<K_{g}<$ $A_{g}^{+}$.

We can see that $f \circ g\left(K_{g}\right)=f\left(K_{g}\right)>g\left(K_{g}\right)=K_{g}$ and $f \circ g\left(A_{f}^{+}\right)=f\left(A_{f}\right)=$ $A_{f}<A_{f}^{+}$. Therefore the map $f \circ g(x)-x$ changes sign on $\left(K_{g}, A_{f}^{+}\right)$. Hence there exists $x \in\left(K_{g}, A_{f}^{+}\right)$such that $f \circ g(x)=x$.

Note that $C_{f g}^{+}<A_{f}^{+}$. To see this fact suppose by contradiction that $C_{f g}^{+} \geq A_{f}^{+}$or equivalently $g^{-1}\left(C_{f}\right) \geq g^{-1}\left(A_{f}\right)$. We know that $C_{f g}^{+}, A_{f}^{+}>C_{g}$ and $g$ is decreasing on $\left(C_{g}, b\right)$. Consequently, applying $g$ in both sides of the last inequality we get $C_{f} \leq A_{f}$ that is impossible. Similarly we prove $C_{g f}^{+}<A_{g}^{+}$.

Since $C_{f g}^{+}<A_{f}^{+}$the carrying capacity of $f \circ g, K_{f g}$, is the greater root of $f \circ g(x)=x$ on $\left(K_{g}, A_{f}^{+}\right)$. We also can see that $g \circ f\left(A_{g}^{+}\right)=g\left(A_{g}\right)<A_{g}^{+}$and $g \circ f\left(A_{g}^{-}\right)=A_{g}>A_{g}^{-}$. So the map $g \circ f(x)-x$ changes sign on $\left(A_{g}^{-}, A_{g}^{+}\right)$and therefore there exists $K_{g f} \in\left(A_{g}^{-}, A_{g}^{+}\right)$such that $g \circ f\left(K_{g f}\right)=K_{g f}$ since $C_{g f}^{+}<A_{g}^{+}$.

Remark 1. Let $f$ and $g$ be two unimodal Allee maps such that $f(x)>g(x)$ for 
all $x$ on $(0, b)$. If $f \circ g\left(C_{g}\right)>C_{g}$ (resp. $g \circ f\left(C_{f}\right)>C_{f}$ ) then the map $f \circ g$ (resp. $g \circ f$ ) has exactly two positives fixed points, the threshold point and the carrying capacity.

Corollary 4.2. Let $f$ and $g$ be two unimodal Allee maps such that $f(x)>g(x)$ for all $x$ on $(0, b)$. If $C_{g}, C_{f}>K_{f}$ then $f \circ g(x)>g \circ f(x), \forall x \in\left[K_{g}, K_{f}\right]$. Moreover, $K_{g}<K_{g f}<K_{f g}<K_{f}$.

Proof. If $C_{g}, C_{f}>K_{f}$ we have that $f$ and $g$ are increasing on $\left[K_{g}, K_{f}\right]$. The composition of increasing maps is an increasing map. The interval $\left[K_{g}, K_{f}\right]$ is invariant under composition because $f \circ g\left(K_{g}\right)>K_{g}, f \circ g\left(K_{f}\right)<K_{f}$ and $g \circ f\left(K_{f}\right)<K_{f}$, $g \circ f\left(K_{g}\right)>K_{g}$. So the map $f \circ g(x)-x$ (resp. $\left.g \circ f(x)-x\right)$ changes sign on $\left[K_{g}, K_{f}\right]$. We know that $f\left(K_{g}\right)>K_{g}$ and therefore $g \circ f\left(K_{g}\right)<f\left(K_{g}\right)=f \circ g\left(K_{g}\right)$ $\left(g(x)<x, \forall x>K_{g}\right)$. On other hand we know that $g\left(K_{f}\right)<K_{f}$ so $f \circ g\left(K_{f}\right)>$ $g\left(K_{f}\right)=g \circ f\left(K_{f}\right)(f(x)>x, \forall x \in] A_{f}, K_{f}[)$. Consequently, $f \circ g(x)>g \circ f(x)$, $\forall x \in\left[K_{g}, K_{f}\right]$. Once that $f \circ g\left(C_{g}\right)<C_{g}$ (resp. $\left.g \circ f\left(C_{f}\right)<C_{f}\right)$ from remark 1 it follows that $K_{f g}$ (resp. $K_{g f}$ ) is the unique fixed point of $f \circ g$ (resp. $g \circ f$ ) on $\left[K_{g}, K_{f}\right]$. The relation order between $K_{f g}$ and $K_{g f}$ is an immediate consequence of the relation order between the composition maps.

COROLlary 4.3. Let $f$ and $g$ be two unimodal Allee maps such that $f(x)>g(x)$ for all $x$ on $(0, b)$. Then following statements holds true

(1) if $C_{f}>K_{f}$ and $C_{g}>K_{g}$ then $K_{f g}, K_{g f} \in\left(K_{g}, K_{f}\right)$.

(2) if $C_{f}<K_{f}$ then we have

a) $K_{f g} \in\left(K_{g}, K_{f}\right)$ if $f \circ g\left(K_{f}\right)<K_{f}$ and $f \circ g\left(C_{f g}^{+}\right)<C_{f g}^{+}$(in the case that $C_{f g}^{+}$doesn't exists we have $K_{f g} \in\left(K_{g}, K_{f}\right)$ if $\left.f \circ g\left(K_{f}\right)<K_{f}\right)$;

b) $K_{f g} \in\left(K_{f}, A_{f}^{+}\right)$if $f \circ g\left(K_{f}\right)>K_{f}$;

c) $K_{g f} \in\left(A_{g}^{-}, K_{g}\right)$ if $g \circ f\left(K_{g}\right)<K_{g}$ and $g \circ f\left(C_{g f}^{+}\right)<C_{g f}^{+}$;

d) $K_{g f} \in\left(K_{g}, K_{f}\right)$ if $g \circ f\left(K_{g}\right)>K_{g}$ and $g \circ f\left(C_{g f}^{+}\right)<C_{g f}^{+}$;

e) $K_{g f} \in\left(C_{g f}^{+}, A_{g}^{+}\right)$if $g \circ f\left(C_{g f}^{+}\right)>C_{g f}^{+}$.

(3) if $C_{f}>K_{f}$ and $C_{g}<K_{g}$ then we have

a) $K_{f g} \in\left(K_{g}, K_{f}\right)$

b) The situation of $K_{g f}$ is similar to (2)c, (2)d and (2)e.

From the previous corollary it is possible, in certain cases, to establish an order relation between the two carrying capacities $K_{f g}$ and $K_{g f}$ of the composition maps $f \circ g$ and $g \circ f$. In particular we are interested in an order when such fixed points are between the carrying capacities of the individual maps. The next result provides this information, respectively.

TheOREM 4.4. Let $f$ and $g$ be two unimodal Allee maps such that $f(x)>g(x)$ for all $x$ on $(0, b)$. Suppose that $C_{f}<K_{f}, C_{g}<K_{g}, f \circ g\left(K_{f}\right)<K_{f}, f \circ g\left(C_{f g}^{+}\right)<C_{f g}^{+}$, $g \circ f\left(K_{g}\right)>K_{g}$ and $g \circ f\left(C_{g f}^{+}\right)>C_{g f}^{+}$. Let $y \in J=\left[k_{g}, k_{f}\right]$ and suppose that $g(y)>K_{f}^{-}, \forall y \in J$, where $K_{f}^{-}$is the left preimage of $K_{f}$ by the map $f$. Then $g \circ f(y)<f \circ g(y), \forall y \in J$. Moreover, $K_{g}<K_{g f}<K_{f g}<K_{f}$.

Proof. Let $K_{f}^{-}$be the left preimage of $K_{f}$ by the map $f$, i.e., $f\left(K_{f}^{-}\right)=K_{f}$. Then $A_{f}<K_{f}^{-}<K_{f}$. Note that $g$ is decreasing on $J=\left[k_{g}, k_{f}\right], g(y)<y, \forall y \in J$ and $f(y)>y, \forall y \in J$.

From the hypothesis we have $g(y)>K_{f}^{-}$and therefore $f \circ g(y)>K_{f}, \forall y \in J$. 
On the other hand $f(y)>K_{f}>y>K_{g}$, and then $g \circ f(y)<f(y)<K_{g}, \forall y \in J$. Consequently, $g \circ f(y)<f \circ g(y), \forall y \in J$.

From the hypothesis of the theorem and remark 1 the theorem is established.

\section{Stability}

The first objective in this section is to formulate in a more precise form definitions of stability in the settings of general periodic difference equations of the form

$$
x_{n+1}=f_{n}\left(x_{n}\right), f_{n+p}=f_{n}, n \in \mathbb{Z}^{+} .
$$

Equivalently, one may speak about the stability in the settings of the nonautonomous periodic dynamical systems $\mathcal{F}=\left\{f_{0}, f_{1}, \ldots, f_{p-1}\right\}$.

Stability notions for periodic difference equations have been investigated by many authors. Including, to cite few, AlSharawi and Angelos [3], AlSharawi, Angelos, Elaydi and Rakesh [4], Henson [11], Yakubu [15], Oliveira and D'Aniello [14], and Selgrado and Roberds [20].

It is our hope that our definitions will standarize the notion and terminology in the area of nonautonomous systems.

Definition 5.1. $\Phi_{i}=f_{i-1} \circ \ldots \circ f_{1} \circ f_{0}$ is called the composition operator with order $i$ associated with Eq. (11) and $\widetilde{\Phi}_{i}=f_{0} \circ f_{1} \circ \ldots \circ f_{i-1}$ is called the reverse composition operator with order $i$ associated with Eq. (11).

Definition 5.2. Let $x^{*}$ be a fixed point of $\mathcal{F}$, i.e., $x^{*}$ is a fixed point of all the members of the set $\mathcal{F}$. Then

(1) $x^{*}$ is stable if given $\varepsilon>0$, there exits $\delta>0$ such that $\left|x_{0}-x^{*}\right|<\delta$ implies $\left|\Phi_{i}\left(x_{0}\right)-x^{*}\right|<\varepsilon, \forall i \geq 1$. Otherwise, $x^{*}$ is unstable.

(2) $x^{*}$ is attracting if there exists $\eta>0$ such that $\left|x_{0}-x^{*}\right|<\eta$ implies $\lim _{n \rightarrow \infty} \Phi_{n}\left(x_{0}\right)=x^{*}$.

(3) $x^{*}$ is asymptotically stable if it is both stable and attracting.

(4) $x^{*}$ is globally asymptotically stable if it is asymptotically stable and $\eta=\infty$.

Many authors use the notion of attractivity of nonhyperbolic fixed point instead of our general definition. The following result provides us the connection between these two notions

Lemma 5.3. Suppose that $\mathcal{F}$ is a set of continuously differentiable maps at $x^{*}$. If $\left|\Phi_{i}^{\prime}\left(x^{*}\right)\right|<1, \forall i \geq 1$ then $x^{*}$ is an asymptotically stable fixed point of $\mathcal{F}$.

Proof. From the hypothesis we have for all $i \geq 1$

$$
\left|\Phi_{i}^{\prime}\left(x^{*}\right)\right|=\left|f_{i-1}^{\prime}\left(x^{*}\right) f_{i-2}^{\prime}\left(x^{*}\right) \ldots f_{1}^{\prime}\left(x^{*}\right) f_{0}^{\prime}\left(x^{*}\right)\right| \leq M<1
$$

Hence there exists an open interval $J=\left(x^{*}-\varepsilon, x^{*}+\varepsilon\right)$ such that $\left|\Phi_{i}^{\prime}(y)\right| \leq M<1$, $\forall y \in J$. By the mean value theorem we know that

$$
\left|\Phi_{1}\left(x_{0}\right)-x^{*}\right|=\left|\Phi_{1}^{\prime}(y)\right|\left|x_{0}-x^{*}\right| \leq M\left|x_{0}-x^{*}\right|,
$$

for any $x_{0} \in I$ and $y$ between $x_{0}$ and $x^{*}$. The last inequality implies that $\Phi_{1}\left(x_{0}\right) \in I$ 
since $M<1$. Using the same argument we get

$$
\left|\Phi_{2}\left(x_{0}\right)-x^{*}\right| \leq M\left|\Phi_{1}\left(x_{0}\right)-x^{*}\right| \leq M^{2}\left|x_{0}-x^{*}\right| .
$$

By mathematical induction, we can prove that

$$
\left|\Phi_{n}\left(x_{0}\right)-x^{*}\right| \leq M^{n}\left|x_{0}-x^{*}\right|, \forall n \geq 1 \text {. }
$$

Assuming $\delta=\varepsilon / 2$, for any $\varepsilon>0$, from $\left|x_{0}-x^{*}\right|<\delta$ follows that $\mid \Phi_{n}\left(x_{0}\right)-$ $x^{*} \mid \leq M^{n} \varepsilon / 2<\varepsilon, \forall n \geq 1$ since $M<1$ and consequently $x^{*}$ is stable. Moreover, $\lim _{n \rightarrow \infty} \Phi_{n}\left(x_{0}\right)=x^{*}$ and thus $x^{*}$ is attracting.

In particular, if $\mathcal{F}$ is a set formed by unimodal Allee maps we have that $x^{*}=0$ is a fixed point of $\Phi_{i}(x)$, for all $i \geq 1$. Since this fixed point is asymptotically stable for each map we have $\left|\Phi_{i}^{\prime}(0)\right|<1$ and thus from the previous lemma $x^{*}=0$ is an asymptotically stable fixed point of $\mathcal{F}$.

Now let us focus our attention on the stability of a periodic cycle for a periodic nonautonomous difference equation. Our definition of stability now follows.

Definition 5.4. Let $C_{r}=\left\{\bar{x}_{0}, \bar{x}_{1}, \ldots, \bar{x}_{r-1}\right\}$ be a $r$-periodic cycle of equation (11) where $f_{n+p}=f_{n}, n \in \mathbb{Z}^{+}, p>1$ and either $r$ divides $p$ or $r$ is a multiple of $p$.

(1) $C_{r}$ is stable if given $\varepsilon>0$ there exists $\delta>0$ such that $\left|x_{0}-\bar{x}_{0}\right|<\delta$ implies $\left|\Phi_{i}\left(x_{0}\right)-\Phi_{i}\left(\bar{x}_{0}\right)\right|<\varepsilon, \forall i \geq 1$. Otherwise, $C_{r}$ is said unstable.

(2) $C_{r}$ is attracting if there exits $\eta>0$ such that $\left|x_{0}-\bar{x}_{0}\right|<\eta$ implies $\lim _{n \rightarrow \infty} \Phi_{r n+i}\left(x_{0}\right)=\bar{x}_{i}, 0 \leq i \leq r-1$.

(3) $C_{r}$ is asymptotically stable if it is both stable and attracting.

(4) $C_{r}$ is globally asymptotically stable if it is asymptotically stable and $\eta=\infty$.

An immediate consequence of this definition now follows

Lemma 5.5. Suppose that $\mathcal{F}=\left\{f_{0}, f_{1}, \ldots, f_{p-1}\right\}$ is a set of continuously differentiable maps at $\bar{x}_{0}$. If $\left|\Phi_{p}^{\prime}\left(\bar{x}_{0}\right)\right|<1$ then the $r$-periodic cycle $C_{r}$ of Eq. (12) is asymptotically stable, where $f_{n+p}=f_{n}, n \in \mathbb{Z}^{+}, p>1$ and either $r$ divides $p$ or $r$ is a multiple of $p$

Proof. The proof is divided into two parts

(1) Consider the first case where $r$ divides $p$, that is, $m r=p$. Suppose that $\left|\Phi_{p}^{\prime}\left(\bar{x}_{0}\right)\right|<1$, i.e.

$$
\left|f_{0}^{\prime}\left(\bar{x}_{0}\right) f_{1}^{\prime}\left(\bar{x}_{1}\right) \ldots f_{r-1}^{\prime}\left(\bar{x}_{r-1}\right) f_{r}^{\prime}\left(\bar{x}_{0}\right) \ldots f_{2 r-1}^{\prime}\left(\bar{x}_{r-1}\right) f_{2 r}^{\prime}\left(\bar{x}_{0}\right) \ldots f_{m r-1}^{\prime}\left(\bar{x}_{r-1}\right)\right| \leq M<1 .
$$

Following the same argument that we the used in the prove of lemma 5.3 we get

$$
\left|\Phi_{r}^{m}\left(x_{0}\right)-\bar{x}_{0}\right| \leq M^{m r}\left|x_{0}-\bar{x}_{0}\right|
$$

This implies that $\lim _{m \rightarrow \infty} \Phi_{r}^{m}\left(x_{0}\right)=\bar{x}_{0}$ since $M<1$. By continuity (the composition of continuous maps is a continuous map) the following statement yields

$$
\Phi_{i} \Phi_{r}^{m}\left(x_{0}\right)=\Phi_{r+i}^{m}\left(x_{0}\right) \underset{m \rightarrow \infty}{\longrightarrow} \Phi_{i}\left(\bar{x}_{0}\right)=\bar{x}_{i}
$$

and thus $C_{r}$ is attracting. Note that $\Phi_{p+i}=\Phi_{m r+i}=\Phi_{i} \Phi_{r}^{m}$. 
To see the stability of $C_{r}$ let us to take $\delta<\varepsilon / 2$, for any given $\varepsilon>0$. Since $\bar{x}_{0} \in C_{r}$ we have $\bar{x}_{0}=\Phi_{r}\left(\bar{x}_{0}\right)=\Phi_{r m}\left(\bar{x}_{0}\right)$ and thus from (13) we have $\left|\Phi_{r m}\left(x_{0}\right)-\Phi_{r m}\left(\bar{x}_{0}\right)\right|<\varepsilon$. Consequently, $\left|\Phi_{i}\left(x_{0}\right)-\Phi_{i}\left(\bar{x}_{0}\right)\right|<\varepsilon, \forall \geq 1$.

(2) In the second case let us to assume that $r=m p$. The dynamics of the points in the $r$-cycle is

$\bar{x}_{1}=\Phi_{1}\left(\bar{x}_{0}\right), \bar{x}_{2}=\Phi_{2}\left(\bar{x}_{0}\right), \ldots, \bar{x}_{p}=\Phi_{p}\left(\bar{x}_{0}\right), \ldots, \bar{x}_{2 p}=\Phi_{p}^{2}\left(\bar{x}_{0}\right), \ldots, \bar{x}_{0}=\Phi_{p}^{m}\left(\bar{x}_{0}\right)$

Following the same argument of the previous case we show that

$$
\left|\Phi_{p}^{n m}\left(x_{0}\right)-\bar{x}_{0}\right|<M^{n m}\left|x_{0}-\bar{x}_{0}\right|
$$

The rest of the prove is similar.

In the particular case, when $\mathcal{F}$ is a periodic set formed by unimodal Allee maps such that $f_{i}<f_{i+1}, \forall i \in\{0,1, \ldots, p\}$, the threshold point $A_{\Phi_{p}}$ of $\Phi_{p}$ is unstable since the map $\Phi_{p}(x)$ is increasing on $\left[0, A_{\Phi_{p}}\right]$ and $\Phi_{p}(x)<x, \forall x \in\left(0, A_{\Phi_{p}}\right)$. The same happens for $\widetilde{\Phi}_{i}$ on $\left[0, A_{\widetilde{\Phi}_{i}}\right]$.

Remark 1. The above theorems cover the hyperbolic case when $\left|\Phi^{\prime}\left(x^{*}\right)\right| \neq 1$. When $\left|\Phi^{\prime}\left(x^{*}\right)\right|=1$ or -1 , the critical point is called neutral. A complete analysis of these nonhyperbolic cases may be found in Elaydi's book "Discrete Chaos" [8].

\section{Bifurcation}

The study of various notions of bifurcation in the setting of nonautonomous systems is still in its infancy stage. The main contribution in this area are the papers by Henson [11], AlSharawi and Angelo [3], and Oliveira and D'Aniello [14]. Our main goal here is to give precise and complete definitions and notions for the various bifurcation notions in the setting of nonautonomous systems. Though our focus here will be on 2 -periodic systems, the ideas presented can be easily extended to the general periodic case.

We start our exposition presenting the following theorem due by Henson [11] where the idea is to perturb the parameters.

TheOREM 6.1. Suppose that $F(\alpha, x): \mathbb{R} \times \mathbb{R} \rightarrow \mathbb{R}$ is non-linear in $x$, one to one in $\alpha, C^{2}$ in both $x$ and $\alpha$, and for a specific real number $\widetilde{\alpha}$, the autonomous difference equation $x_{n+1}=F\left(\widetilde{\alpha}, x_{n}\right)$ has an attracting $r$-periodic cycle $\left\{\bar{x}_{0}, \bar{x}_{1}, \ldots, \bar{x}_{r-1}\right\}$, with a minimal period $r$. Then for sufficiently small $\epsilon>0$, if $\alpha_{0}, \alpha_{1}, \ldots, \alpha_{p-1} \in$ $(\widetilde{\alpha}-\epsilon, \widetilde{\alpha}+\epsilon)$ then the $p-$ periodic difference equation $x_{n+1}=F\left(\alpha_{n}, x_{n}\right), \alpha_{n+p}=\alpha_{n}$ has $t$ attracting periodic solutions of minimal period $s$, where $t=\operatorname{gcd}(p, r)$ (greatest common divisor of $p$ and $r$ ), and $s=l c m(p, r)$ (least common multiple of $p$ and $r)$.

Proof. See [11]

Consider the 2 -periodic system $\mathcal{F}=\left\{f_{0}, f_{1}\right\}, f_{0} \neq f_{1}$, where both maps arise from a one-parameter family of maps $f_{\alpha}$ in which $f_{0}=f_{\alpha_{0}}$ and $f_{1}=f_{\alpha_{1}}$. Let $C_{r}=$ $\left\{\bar{x}_{0}, \bar{x}_{1}, \ldots, \bar{x}_{r-1}\right\}$ be an $r$-periodic cycle of $f_{\widetilde{\alpha}}$. In the following table we summarize the ideas presents in theorem 6.1 for sufficiently small $\epsilon$ and $\alpha_{0}, \alpha_{1} \in(\widetilde{\alpha}-\epsilon, \widetilde{\alpha}+\epsilon)$. 


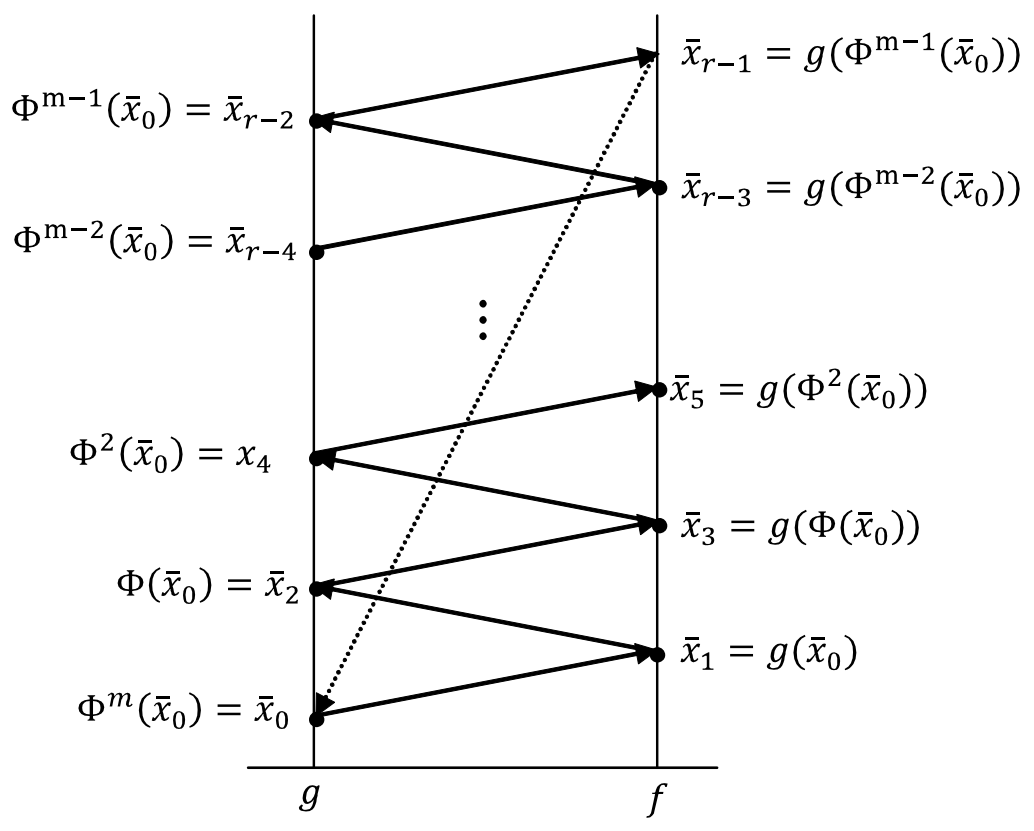

Figure 3. Sequence of the periodic points $\left\{\bar{x}_{0}, \bar{x}_{1}, \ldots, \bar{x}_{r-1}\right\}$ in the fibers $g$ and $f$

\begin{tabular}{c|c|c|l}
\hline \hline$r$ & $\operatorname{gcd}(r, 2)$ & $l c m(r, 2)$ & Conclusion \\
\hline 1 & 1 & 2 & $\mathcal{F}$ has one G.A.S. 2-periodic cycle \\
\hline 2 & 2 & 2 & $\mathcal{F}$ has two A.S. 2-periodic cycle \\
\hline 4 & 2 & 4 & $\mathcal{F}$ has two A.S. 4-periodic cycle \\
\hline$\vdots$ & $\vdots$ & $\vdots$ & $\vdots$ \\
\hline $2 m$ & 2 & $2 m$ & $\mathcal{F}$ has two A.S. $2 m$ - periodic cycle \\
\hline \hline
\end{tabular}

Let $r=2 m, m \geq 1$. With $\Phi_{2}=f \circ g$, one may write the orbit of $C_{r}$ as (see figure 3)

$$
\mathcal{O}\left(\bar{x}_{0}\right)=\left\{\bar{x}_{0}, g\left(\bar{x}_{0}\right), \Phi_{2}\left(\bar{x}_{0}\right), g \circ \Phi_{2}\left(\bar{x}_{0}\right), \Phi_{2}^{2}\left(\bar{x}_{0}\right), \ldots, \Phi_{2}^{m-1}\left(\bar{x}_{0}\right), g \circ \Phi_{2}^{m-1}\left(\bar{x}_{0}\right)\right\}
$$

or equivalently

$\mathcal{O}\left(\bar{x}_{1}\right)=\left\{f \circ \widetilde{\Phi}_{2}^{m-1}\left(\bar{x}_{1}\right), \bar{x}_{1}, f\left(\bar{x}_{1}\right), \widetilde{\Phi}_{2}\left(\bar{x}_{1}\right), f \circ \widetilde{\Phi}_{2}\left(\bar{x}_{1}\right), \ldots, f \circ \widetilde{\Phi}_{2}^{m-2}\left(\bar{x}_{1}\right), \widetilde{\Phi}_{2}^{m-1}\left(\bar{x}_{1}\right)\right\}$

where $\widetilde{\Phi}_{2}=g \circ f$. Hence the order of the composition is irrelevant to the dynamics of the system.

In the sequel, we assume that the maps $f$ and $g$ arise from a one-parameter family of maps such that $f=f_{\alpha}$ and $g=g_{\beta}$ with $\beta=q \alpha$ for some real number $q>0$. Thus one may write, without loss of generality, our system as $\mathcal{F}=\left\{f_{\alpha}, g_{\alpha}\right\}$.

The dynamics of $\mathcal{F}$ depends very much on the parameter and the qualitative structure of the dynamical system changes as the parameter changes. These qualitative changes in the dynamics of the system are called bifurcation and the parameter values at which they occur are called bifurcation points. For autonomous systems or single maps the bifurcation analysis may be found in Elaydi [8].

In a one-dimensional systems generated by a one-parameter family of maps $f_{\alpha}$, bifurcation at a fixed point $x^{*}$ occurs when $\frac{\partial f}{\partial x}\left(\alpha^{*}, x^{*}\right)=1$ or -1 at a bifurcation point $\alpha^{*}$. The farmer case leads to a saddle-node bifurcation, while the latter case 
leads to a period-doubling bifurcation.

Our objective here is to extend this analysis to 2 -periodic difference equations or $\mathcal{F}=\left\{f_{\alpha}, g_{\alpha}\right\}$. To simplify the notation we write $\Phi$ instead of $\Phi_{2}$, and we write $\Phi(\alpha, x)$ instead of $\Phi(x)$. Let $C_{r}=\left\{\bar{x}_{0}, \bar{x}_{1}, \ldots, \bar{x}_{r-1}\right\}$ be a $r$-periodic cycle of $\mathcal{F}$ and suppose that $2 m=r, \Phi=f \circ g$ and $\widetilde{\Phi}=g \circ f$. Then $\Phi^{m}\left(\bar{x}_{2 i}\right)=\bar{x}_{(2 i) m o d r}$ and $\widetilde{\Phi}^{m}\left(\bar{x}_{2 i+1}\right)=\bar{x}_{(2 i+1) \operatorname{modr}}, 1 \leq i \leq m$. In general we have $\Phi^{n m}\left(\bar{x}_{2 i}\right)=\bar{x}_{(2 i) \operatorname{modr}}$ and $\widetilde{\Phi}^{n m}\left(\bar{x}_{2 i+1}\right)=\bar{x}_{(2 i+1) \operatorname{modr}}, n \geq 1$.

Assuming $\frac{\partial \Phi^{m}}{\partial x}\left(\bar{\alpha}, \bar{x}_{0}\right)=1$ at a bifurcation point $\bar{\alpha}$ by the chain rule, we have

$$
\frac{\partial \Phi}{\partial x}\left(\bar{\alpha}, \bar{x}_{2 m-2}\right) \frac{\partial \Phi}{\partial x}\left(\bar{\alpha}, \bar{x}_{2 m-4}\right) \ldots \frac{\partial \Phi}{\partial x}\left(\bar{\alpha}, \bar{x}_{2}\right) \frac{\partial \Phi}{\partial x}\left(\bar{\alpha}, \bar{x}_{0}\right)=1
$$

or

$$
f_{\bar{\alpha}}^{\prime}\left(\bar{x}_{2 m-1}\right) g_{\bar{\alpha}}^{\prime}\left(\bar{x}_{2 m-2}\right) f_{\bar{\alpha}}^{\prime}\left(\bar{x}_{2 m-3}\right) g_{\bar{\alpha}}^{\prime}\left(\bar{x}_{2 m-4}\right) \ldots f_{\bar{\alpha}}^{\prime}\left(\bar{x}_{3}\right) g_{\bar{\alpha}}^{\prime}\left(\bar{x}_{2}\right) f_{\bar{\alpha}}^{\prime}\left(\bar{x}_{1}\right) g_{\bar{\alpha}}^{\prime}\left(\bar{x}_{0}\right)=1
$$

Applying $g_{\bar{\alpha}}$ on both sides of the identity $\Phi^{m}\left(\bar{\alpha}, \bar{x}_{0}\right)=\bar{x}_{0}$, yields $\widetilde{\Phi}^{m}\left(\bar{\alpha}, \bar{x}_{1}\right)=\bar{x}_{1}$. Differentiating both sides of this equation we get

$$
\frac{\partial \widetilde{\Phi}}{\partial x}\left(\bar{\alpha}, \bar{x}_{2 m-1}\right) \frac{\partial \widetilde{\Phi}}{\partial x}\left(\bar{\alpha}, \bar{x}_{2 m-3}\right) \ldots \frac{\partial \widetilde{\Phi}}{\partial x}\left(\bar{\alpha}, \bar{x}_{3}\right) \frac{\partial \widetilde{\Phi}}{\partial x}\left(\bar{\alpha}, \bar{x}_{1}\right)=1
$$

or equivalently

$$
g_{\bar{\alpha}}^{\prime}\left(\bar{x}_{0}\right) f_{\bar{\alpha}}^{\prime}\left(\bar{x}_{2 m-1}\right) g_{\bar{\alpha}}^{\prime}\left(\bar{x}_{2 m-2}\right) f_{\bar{\alpha}}^{\prime}\left(\bar{x}_{2 m-3}\right) \ldots g_{\bar{\alpha}}^{\prime}\left(\bar{x}_{4}\right) f_{\bar{\alpha}}^{\prime}\left(\bar{x}_{3}\right) g_{\bar{\alpha}}^{\prime}\left(\bar{x}_{2}\right) f_{\bar{\alpha}}^{\prime}\left(\bar{x}_{1}\right)=1 .
$$

Hence Eq. (17) is equivalent to Eq. (18). More generally the following the relation yields

$$
\frac{\partial \Phi^{m}}{\partial x}\left(\bar{\alpha}, \bar{x}_{2 j}\right)=\frac{\partial \widetilde{\Phi}^{m}}{\partial x}\left(\bar{\alpha}, \bar{x}_{2 j-1}\right), j \in\{0,1, \ldots, m-1\}
$$

The next result gives conditions for the saddle-node bifurcation.

Theorem 6.2 Saddle-node Bifurcation $\quad$ Let $C_{r}=\left\{\bar{x}_{0}, \bar{x}_{1}, \ldots, \bar{x}_{r-1}\right\}$ be a $r$-periodic cycle of $\mathcal{F}$. Suppose that both $\frac{\partial^{2} \Phi}{\partial x^{2}}$ and $\frac{\partial^{2} \Phi}{\partial^{2}}$ exist and are continuous in a neighbourhood of a periodic orbit such that $\frac{\partial \Phi^{m}}{\partial x}\left(\bar{\alpha}, \bar{x}_{0}\right)=1$ for the periodic point $\bar{x}_{0}$. Assume also that

$$
A=\frac{\partial \Phi^{m}}{\partial \alpha}\left(\bar{\alpha}, \bar{x}_{0}\right) \neq 0 \text { and } B=\frac{\partial^{2} \Phi^{m}}{\partial x^{2}}\left(\bar{\alpha}, \bar{x}_{0}\right) \neq 0
$$

Then there exists an interval $J$ around the periodic orbit and a $C^{2}-$ map $\alpha=h(x)$, where $h: J \rightarrow \mathbb{R}$ such that $h\left(\bar{x}_{0}\right)=\bar{\alpha}$, and $\Phi^{m}(x, h(x))=x$. Moreover, if $A B<0$, the periodic points exists for $\alpha>\bar{\alpha}$, and, if $A B>0$, the periodic points exists for $\alpha<\bar{\alpha}$.

Proof. The proof is similar to the proof of theorem 2.5 in $[8$, pp. 86] and will be omitted.

When $\frac{\partial \Phi^{m}}{\partial x}\left(\bar{\alpha}, \bar{x}_{0}\right)=1$ but $\frac{\partial \Phi^{m}}{\partial \alpha}(\bar{\alpha}, \bar{x})=0$, two types of bifurcation appear. The first is called transcritical bifurcation which appears when $\frac{\partial^{2} \Phi^{m}}{\partial x^{2}}\left(\bar{\alpha}, \bar{x}_{0}\right) \neq 0$ and the second called pitchfork bifurcation which appears when $\frac{\partial^{2} \Phi^{m}}{\partial x^{2}}\left(\bar{\alpha}, \bar{x}_{0}\right)=0$ (see 
table 2.1 in [8, pp. 90]. In [14], the authors studied the pitchfork bifurcation for 2 -periodic systems in which the maps have negative Schwarzian derivative.

In the next result we characterize period-doubling bifurcation.

Theorem 6.3 Period-Doubling Bifurcation Let $C_{r}=\left\{\bar{x}_{0}, \bar{x}_{1}, \ldots, \bar{x}_{r-1}\right\}$ be a $r$-periodic cycle of $\mathcal{F}$. Assume that both $\frac{\partial^{2} \Phi}{\partial x^{2}}$ and $\frac{\partial \Phi}{\partial \alpha}$ exist and are continuous in a neighbourhood of a periodic orbit, $\frac{\partial \Phi^{m}}{\partial x}\left(\bar{\alpha}, \bar{x}_{0}\right)=-1$ for the periodic point $\bar{x}_{0}$ and $\frac{\partial^{2} \Phi^{2 m}}{\partial \alpha \partial x}\left(\bar{\alpha}, \bar{x}_{0}\right) \neq 0$. Then, there exists an interval $J$ around the periodic orbit and a function $h: J \rightarrow \mathbb{R}$ such that $\Phi^{m}(x, h(x)) \neq x$ but $\Phi^{2 m}(x, h(x))=x$.

Proof. The proof is similar to the proof of the theorem 2.7 in [8, pp 89] and will be omitted.

Note that if $\mathcal{W}$ is a periodic set formed by unimodal Allee maps, neither the zero fixed point nor the threshold point can contribute to bifurcation, since the former is always asymptotically stable and the latter is always unstable. Hence bifurcation may only occur at the carrying capacity of $\mathcal{W}$.

Now we are going to apply the above results to study the bifurcation of the system $\mathcal{W}=\left\{f_{0}, f_{1}\right\}$, where $f_{i}(x)=a_{i} x^{2}(1-x), i=0,1, x \in[0,1]$ and $a_{i}>0$.

For an individual map $g(x)=a x^{2}(1-x)$, the dynamics is interesting but predictable. For $a<4$ we have a globally asymptotically stable zero fixed point and no other fixed point. A new unstable fixed point born at $a=4$ after which $g(x)$ becomes a unimodal map with an Allee effect. Henceforth, we assume that $a>4$.

To determine the two main types of bifurcation, we solve the equations

$$
\left\{\begin{array}{l}
\bar{x}_{0}=f_{1}\left(f_{0}\left(\bar{x}_{0}\right)\right) \\
f_{1}^{\prime}\left(F_{0}\left(\bar{x}_{0}\right)\right) f_{0}^{\prime}\left(\bar{x}_{0}\right)=1
\end{array}\right.
$$

and

$$
\left\{\begin{array}{l}
\bar{x}_{0}=f_{1}\left(f_{0}\left(\bar{x}_{0}\right)\right) \\
f_{1}^{\prime}\left(f_{0}\left(\bar{x}_{0}\right)\right) f_{0}^{\prime}\left(\bar{x}_{0}\right)=-1
\end{array}\right.
$$

Using the command "resultant" in Mathematica or Maple Software, we eliminate the variable $\bar{x}_{0}$ in both systems. Eq 20 yields

$$
\begin{gathered}
16777216+16384 a_{0} a_{1}-576000 a_{0}^{2} a_{1}+84375 a_{0}^{3} a_{1}-576000 a_{0} a_{1}^{2}+914 a_{0}^{2} a_{1}^{2}- \\
350 a_{0}^{3} a_{1}^{2}+84375 a_{0} a_{1}^{3}-350 a_{0}^{2} a_{1}^{3}+19827 a_{0}^{3} a_{1}^{3}-2916 a_{0}^{4} a_{1}^{3}-2916 a_{0}^{3} a_{1}^{4}+432 a_{0}^{4} a_{1}^{4}=0
\end{gathered}
$$

while Eq. 21 yields

$$
\begin{gathered}
100000000-120000 a_{0} a_{1}-2998800 a_{0}^{2} a_{1}+453789 a_{0}^{3} a_{1}-2998800 a_{0} a_{1}^{2}-4598 a_{0}^{2} a_{1}^{2}+ \\
2702 a_{0}^{3} a_{1}^{2}+453789 a_{0} a_{1}^{3}+2702 a_{0}^{2} a_{1}^{3}+89765 a_{0}^{3} a_{1}^{3}-13500 a_{0}^{4} a_{1}^{3}-13500 a_{0}^{3} a_{1}^{4}+2000 a_{0}^{4} a_{1}^{4}=0
\end{gathered}
$$

For each one of these two equations we invoke the implicit function theorem to plot, in the $\left(a_{0}, a_{1}\right)$-plane, the bifurcation curves (see figure 4 ).

The black curves are the solutions of the first equation, while the grey curves are the solutions of the second equation. For values of $a_{0}$ and $a_{1}$ in region A, the fixed point $x^{*}=0$ of $\mathcal{W}$ is globally asymptotically stable. For values of $a_{0}, a_{1} \in(4,5.27)$, approximately, in the region $\mathrm{B}$, we have one globally asymptotically stable 2-periodic cycle. Moreover, in the same region B and for values 


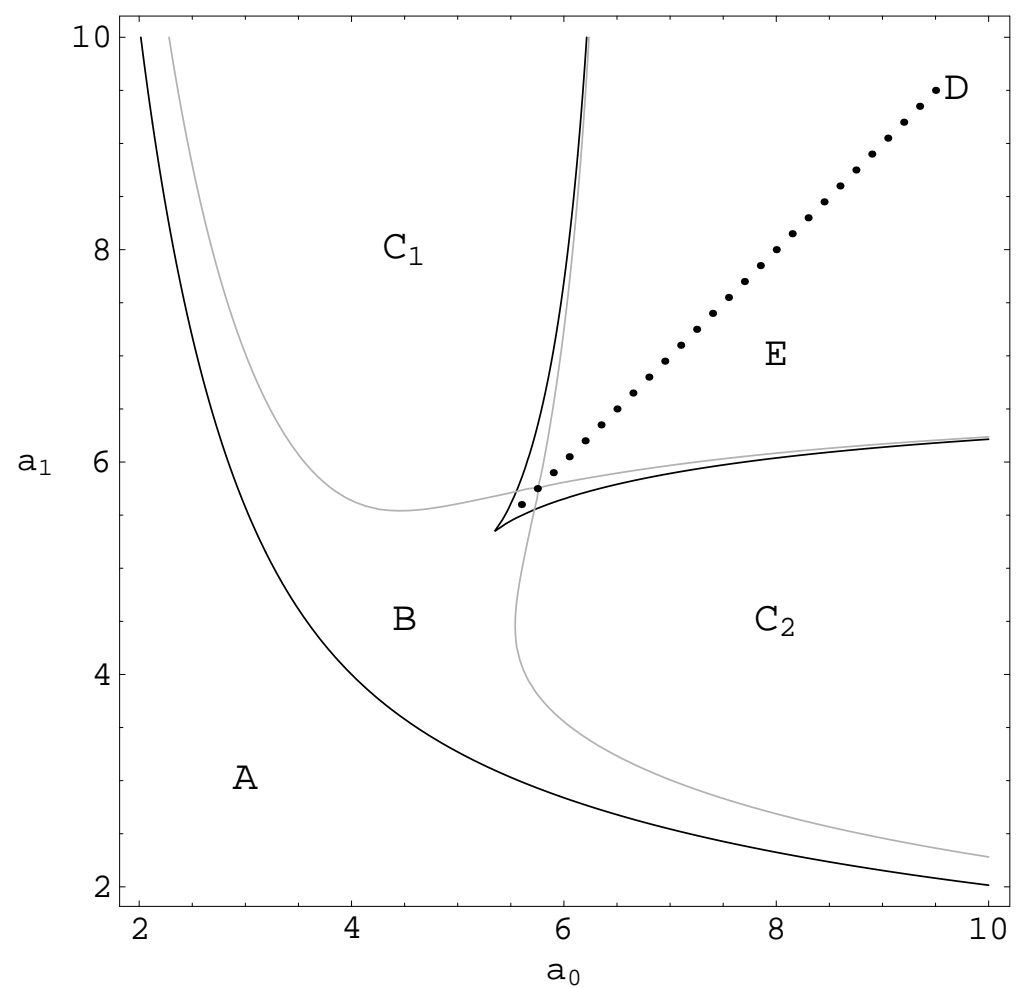

Figure 4. Bifurcations curves for the 2-periodic nonautonomous difference equation with Allee effects $x_{n+1}=a_{n} x_{n}^{2}\left(1-x_{n}\right), a_{n+2}=a_{n}$ and $x_{n+2}=x_{n}$ in the $\left(a_{0}, a_{1}\right)$-plane

$a_{0}, a_{1} \in(5.27,5.6869003)$, approximately, system $\mathcal{W}$ has two asymptotically stable 2 -periodic cycles.

Computation shows that the hypotheses of theorem 6.2 are satisfied when $a_{0}$ and $a_{1}$ belong to a curve between A and B. Consequently, this curve is the Saddle-node curve. The black cusp is the pitchfork bifurcation curve. In region $\mathrm{D}$, system $\mathcal{W}$ has two asymptotically stable $2-$ periodic cycles.

The grey curves are where the period-doubling occurs. Therefore, for certain values of the parameters $a_{0}$ and $a_{1}$ in regions $C_{1}, C_{2}$, and $E$, the system $\mathcal{W}$ has two asymptotically stable 4 -periodic cycles. One rose from the period-doubling and the other from the pitchfork bifurcation.

\section{References}

[1] W. C. Allee, The Social Life of Animals, William Heinemann, London 1938.

[2] L. Allen, J. Fagan, G. Hognas, and H. Fagerholm, Population extinction in discrete-time stochastic population models with an Allee effect, Journal of Difference Equations and Applications, Vol. 11, No. 4-5 (2005), pp. 273-293.

[3] Z. AlSharawi and J. Angelos, On the periodic logistic equation, Appl. Math. Comput., Vol. 180, No 1, pp. 342-352, 2006.

[4] Z. AlSharawi, J. Angelos, S. Elaydi and L. Rakesh, An extension of Sharkovsky's theorem to periodic difference equations, J. Math. Anal. Appl., J. Math. Anal. Appl., Vol. 316, 128-141, 2006.

[5] F. Courchamp, L. Berec, and J. Gascoigne, Allee effects in Ecology and Conservation, Oxford University Press, 2008.

[6] B. Dennis, Allee effects: population growth, critical density, and the chance of extinction, Nat. Res. Model., 3, pp. 481-538, 1989.

[7] J. M. Cushing, Oscillations in age-structured population models with an Allee effect, Journal of Computational and Applied Mathematics 52, 71-80, 1994.

[8] S. Elaydi, Discrete Chaos: With Applications in Science and Engineering, Chapman and Hall/CRC, Second Edition, 2008.

[9] S. Elaydi, and R. Sacker, Skew-product dynamical systems: Applications to difference equations, Proceedings of the Second Annual Celebration of Mathematics, United Arab Emirates, 2005.

[10] S. Elaydi, and R. Sacker, Global stability of periodic orbits of nonautonomous difference equations and population biology, Journal of Differential Equations, Vol. 208, pp. 258-273 (2005). 
[11] S. Henson, Multiple attractors an resonance in periodically forced population models, Physica D. 140, pp. 33-49, 2000

[12] J. Li, B. Song and X. Wang, An extended discrete Ricker population model with Allee effects, Journal of Difference Equations and Applications, Vol. 13, No. 4 (2007), pp. 309-321.

[13] R. Luís, S.Elaydi and H. Oliveira, An economic model with Allee effect, Journal of Difference Equations and Applications, to appear.

[14] H. Oliveira and E. D'Aniello, Pitchfork bifurcation for non autonomous interval maps, Journal of Difference Equations and Applications, to appear.

[15] A. Yakubu, Multiple Attractors in Juvenile-adult Single Species Models, J. Differ. Equ. Appl., Vol 9 , No. 12 (2003), pp. 1083-1098.

[16] A. Yakubu, Allee effects in a discrete-time SIS epidemic model with infected newborns, J. Differ. Equ. Appl. Vol 13, No. 4 (2007), pp. 341-356.

17] R. Sacker and S. Elaydi, Population models with Allee effects: A new model, in progress, 2008.

[18] R. Sacker and J. Sell, Lifting properties in skew-product flows with applications to Differential Equations, AMS Memoirs, Vol. 11, No. 190, 1977

[19] S. Schreiber, Allee effects, extinctions, and chaotic transients in simple population models, Theoretical Populations Biology, 64 (2003), 201-209.

[20] J. Selgrade and H. Roberds, On the structure of attractors for discrete periodically forced systems with applications to populations models, Physica D., 158, 69-82, 2001.

[21] R. Sophia and J. Jang, Allee effects in a discrete-time host-parasitoid model, Journal of Difference Equations and Applications, Vol. 12, No. 2 (2006), 165-181. 